\title{
Gluteal hidradenitis suppurativa presenting pemphigus-like findings: case report
}

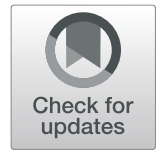

\author{
Yuichi Kurihara $^{1^{*}}$ (D) and Masutaka Furue ${ }^{2}$
}

\begin{abstract} hidradenitis suppurativa is rare.

\section{Background}

Hidradenitis suppurativa is one member of the follicular occlusion triad: acne conglobata, hidradenitis suppurativa, and dissecting cellulitis of the scalp [1]. This group of conditions presents with deep scarring folliculitis composed of multichannel draining sinus and abscesses [1]. Hidradenitis suppurativa is a progressive inflammatory disease and emerges comorbid autoimmune disease [2]. We report a rare case of gluteal hidradenitis suppurativa which also presented acantholysis and desmoglein autoantibodies.
\end{abstract}

Background: Hidradenitis suppurativa is one member of the follicular occlusion triad: acne conglobata, hidradenitis suppurativa, and dissecting cellulitis of the scalp. The presence of acantholysis and desmoglein autoantibodies in

Case presentation: We report a case of 68-year-old male with a diagnosis of gluteal hidradenitis suppurativa copresenting pemphigus-like findings including acantholysis and positive desmoglein autoantibodies.

Conclusion: To our knowledge, comorbidity of gluteal hidradenitis suppurativa and pemphigus-like findings has not been reported before. This case implies a relationship between two different conditions; the follicular occlusion triad and pemphigus, highlighting a potential induction of pemphigus-like lesion by chronic inflammatory process.

Keywords: Hidradenitis suppurativa, Pemphigus, Acantholysis, Desmoglein autoantibodies

\section{Case presentation}

A 68-year-old male presented slightly tender brown nodules and purulent discharge on the right thigh. There were no evident blisters or erosions. He had first developed these lesions three years previously and they had been gradually enlarged. Physical examination revealed brown nodules, multiple fistulae, and scars on the right side of the thigh (Fig. 1a). No other areas of the body including mucosal areas were affected. The patient did not report any gastrointestinal symptoms and had no remarkable past medical history including immunodeficiency and relevant family history. Staphylococcus aureus was cultured from the lesion

\footnotetext{
* Correspondence: yukuri549@gmail.com

${ }^{1}$ Department of Dermatology, Steel Memorial Yawata Hospital, 1-1-1

Harunomachi, Yahatahigashi-ku, Kitakyushu 805-8508, Japan

Full list of author information is available at the end of the article
}

but oral antibiotic treatment for over three months was ineffective, then surgical removal of the lesion was performed. The whole lesion had been totally excised and processed for formalin-fixation. Therefore, standard direct immunofluoresence test was not performed. Indirect immunofluorescence finding was negative. The results of serum ELISA tests for anti-desmoglein 1 and 3 (MESACUP desmoglein 1 and desmoglein 3, MBL) were positive (ELISA titers were 36 and 11 , respectively) and epidermal hyperplasia, numerous intraepidermal clefts and acantholysis were demonstrated in histology (Fig. 1b, c). Furthermore, the findings of gluteal hidradenitis suppurativa, namely, multiple sinus tracts, abscesses, chronic inflammation with polymorphonuclear leukocytes and lymphocytes, and follicle-based inflammation were observed (Fig. 1d, e). Considering the age of onset, unilaterality of lesion and the site and extent of lesion, hidradenitis suppurativa was compatible rather than acne conglobata. At the time of writing, no signs of recurrence after the surgical treatment have emerged.

The differential diagnosis includes pyoderma vegetans, pemphigus vegetans, pyodermatitis-pyostomatitis and other autoimmune blistering dermatoses. In the present case, the absence of mucosal lesions, abdominal symptoms, and eosinophilia ruled out the diagnosis of pyoderma vegetans and pyodermatitis-pyostomatitis. The response to oral antibiotics was poor and ruled out infected pemphigus 


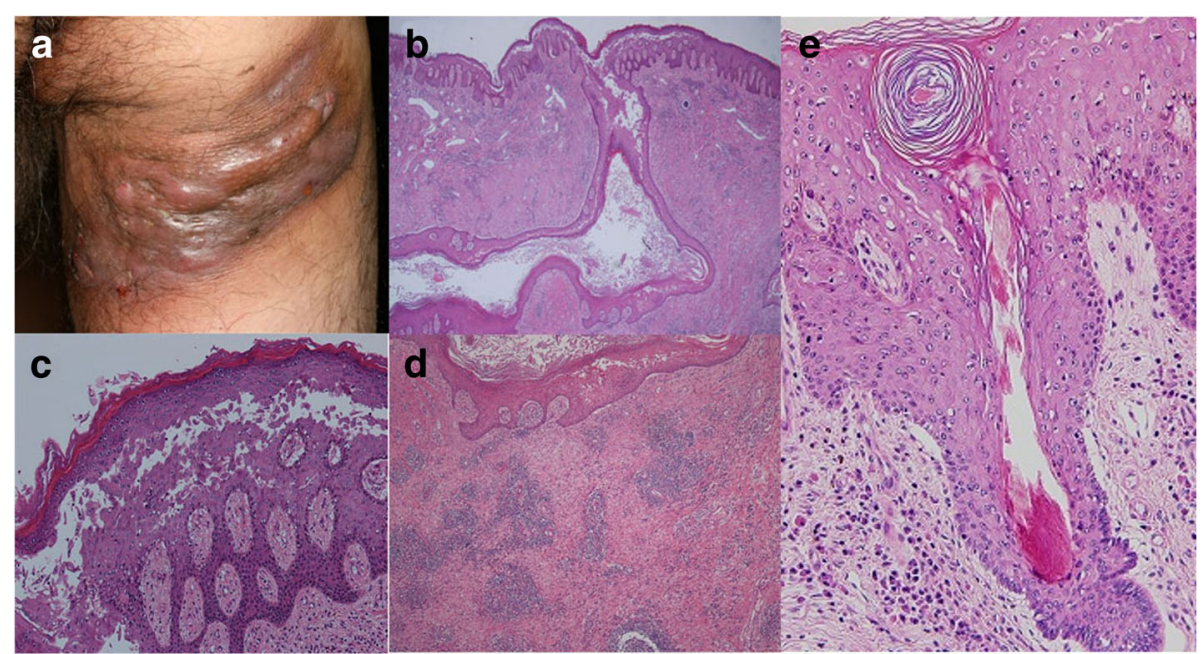

Fig. 1 (a) Brown nodules, multiple fistula and scars on the right side of the thigh. (b) Clefts and acantholysis in the epidermis (hematoxylin and eosin, original magnification $\times 40$ ). (c) Presence of intraepidermal acantholytic cells (original magnification $\times 200$ ) (d) Abscesses and chronic inflammation in the upper dermis. (hematoxylin and eosin, original magnification $\times 100$ ). (e) Involvement of hair follicle in the lesion (original magnification $\times 200$ )

vegetans. We diagnosed this case as hidradenitis suppurativa with pemphigus-like findings because of the unilaterally-localized lesion, low titer of desmoglein antibodies and negative indirect immunofluorescence finding, although concomitant presence of hidradenitis suppurativa and pemphigus could not be ruled out exactly.

\section{Discussion and conclusion}

Although the precise pathogenesis is not fully understood, a previous study described the pemphigus-pyoderma spectrum [3]. The present case also suggests a potential (at least coincidental) relationship between the follicular occlusion triad and pemphigus-like findings. Interestingly, although the blood test results were positive for IgG antibody to desmoglein 1 and 3, the lesion was confined to the thigh. A previous report indicated the association between recurrent staphylococcal infection and the development of an anti-desmoglein 3 antibody response $[4,5]$. It was possible that recurrent bacterial infections may influence or aggravate the intraepidermal lesions in the present case. Finally, the present case may highlight a possibility that pemphigus-like findings can be triggered or induced by chronic inflammatory process like hidradenitis supprativa.

\section{Acknowledgements}

Not applicable.

\section{Authors' contributions}

YK was involved in the conception of the work, the acquisition of data and drafted the work. MF was involved in the design of the work, the analysis of data and revising the manuscript. All authors have read and approved the manuscript.

\section{Funding}

This work was supported partly by grants from The Ministry of Health, Labor, and Welfare and The Ministry of Education, Culture, Sports, Science, and
Technology, Japan. The funder supported data analysis and had no role in design of the work, data collection, and preparation of the manuscript.

Availability of data and materials

All data is included within the published article.

Ethics approval and consent to participate Not applicable.

\section{Consent for publication}

The patient gave written informed consent to the publication of the case report, including clinical details and images. A copy of the written informed consent is available for review by the Editor of this journal if requested.

\section{Competing interests}

The authors declared that they have no competing interests.

\section{Author details}

${ }^{1}$ Department of Dermatology, Steel Memorial Yawata Hospital, 1-1-1 Harunomachi, Yahatahigashi-ku, Kitakyushu 805-8508, Japan. ${ }^{2}$ Department of Dermatology, Graduate School of Medical Sciences, Kyushu University, 3-1-1 Maidashi, Higashi-ku, Fukuoka 812-8582, Japan.

Received: 20 February 2019 Accepted: 16 July 2019

Published online: 23 July 2019

\section{References}

1. Spitzer I. Dermatitis follicularis et perifollicularis conglobata. Dermatol Z. 1901;10:8.

2. Kohorst JJ, Kimball AB, Davis MD. Systemic associations of hidradenitis suppurativa. J Am Acad Dermatol. 2015;73:S27-35.

3. Jacyk WK. Treatment of pyoderma vegetans of Hallopeau with dapsone. Arch Dermatol. 1979:115:1035-6.

4. Ayari H. Recurrent staphylococcal infection of the face associated with the development of anti-Dsg3 antibody response. Ann Biol Clin. 2011;69:577-9.

5. Dar SA, Das S, Bhattacharya SN, et al. Possible role of superantigens in inducing autoimmunity in pemphigus patients. J Dermatol. 2011;38:980-7.

\section{Publisher's Note}

Springer Nature remains neutral with regard to jurisdictional claims in published maps and institutional affiliations. 